\title{
Nasal nitric oxide in man
}

\author{
J O N Lundberg, E Weitzberg
}

The past decade has witnessed an explosion in the interest of biologists in the gas nitric oxide (NO). This highly reactive free radical, first considered only a noxious air pollutant, is produced in mammalian cells by specific enzymes and is believed to play a vital role in many biological events including regulation of blood flow, platelet function, immunity, and neurotransmission. ${ }^{12}$ Direct measurement of $\mathrm{NO}$ in biological tissues is difficult to perform because this gas reacts rapidly with, for example, haemoglobin or other $\mathrm{Fe}^{2+}-$ containing proteins. It is therefore often necessary to rely on indirect measurements in order to detect NO synthesis in vivo. Unlike the situation in most biological tissues where $\mathrm{NO}$ is rapidly destroyed, in the gas phase NO is fairly stable at low concentrations. ${ }^{3} \mathrm{NO}$ produced in superficial structures of hollow organs will diffuse into the lumen and thus be detectable in gas collected from such organs.

The presence of NO in exhaled breath of humans was first demonstrated by Gustafsson et al in $1991 .{ }^{4}$ Later studies clearly showed that, in healthy controls at rest, almost all NO found in exhaled air originates from the upper airways with only a minor contribution from the lower respiratory tract and the lungs. ${ }^{5-7}$

\section{Origin of nasal NO}

The exact origin of the NO found in nasal air and the relative contribution from different sources within the nasal airways are not known. There are, however, some indications that favour the paranasal sinuses rather than the mucosa of the nasal cavity as a major source of nasal NO in adult healthy humans. ${ }^{8}$ Firstly, NO release in the sinuses is markedly reduced by intrasinus instillation of an $\mathrm{NO}$ synthase inhibitor (L-NAME) whereas nasal NO concentrations are only slightly reduced following intranasal administration of this inhibitor. ${ }^{9}$ Secondly, immunohistochemical and in situ hybridisation studies showed dense staining for nitric oxide synthase (NOS) and its mRNA in sinus epithelium whereas only weak staining was found in the nasal epithelium. ${ }^{9}$ Thirdly, a transient decrease in nasal NO (measured from one nostril) was observed in a study when air was continuously removed from one maxillary sinus during nasal sampling and the opposite (transient elevation) occurred when air was injected into the same sinus. ${ }^{10}$ Fourthly, nasal NO levels are markedly reduced in patients who generally have mucus filled paranasal sinuses and obstructed sinus ostia (Kartagener's syndrome, ${ }^{6}$ cystic fibrosis, ${ }^{11}$ acute sinusitis $\left.^{12}\right)$. Finally, nasal NO levels are high in humans and other primates ${ }^{13}$ but very low in baboons,${ }^{14}$ the only mammal lacking paranasal sinuses.
Both immunohistochemical and in situ hybridisation studies indicate that the NOS found in healthy sinus epithelium ${ }^{9}$ is identical or very closely related to the inducible NOS (iNOS) that has been cloned from activated human hepatocytes. ${ }^{15}$ Moreover, NOS activity in sinus mucosa is predominantly calcium independent, a characteristic associated with iNOS ${ }^{16}$ However, the regulation of the expression and the activity of sinus NOS seems to differ from that previously described for iNOS. Thus, sinus NOS is constantly expressed and seems to be resistant to steroids, ${ }^{9}{ }^{16}$ properties normally associated with the low rate NO producing endothelial and neuronal NOS. Immunostaining for NOS in the nasal mucosa has shown only weak staining for iNOS. ${ }^{9}$ Moreover, Ramis et al found only calcium dependent NOS activity in nasal mucosa from healthy subjects. ${ }^{17}$ Human nasal epithelium from patients with nasal polyp disease express increased iNOS mRNA compared with normal epithelium. ${ }^{18}$

\section{Measurement of nasal NO}

Almost all studies in the literature on the measurement of nasal NO have used the chemiluminescence method. This method uses an excess of ozone $\left(\mathrm{O}_{3}\right)$ which reacts with $\mathrm{NO}$ to produce nitric dioxide $\left(\mathrm{NO}_{2}\right)$ with an electron in an excited stage $\left(\mathrm{NO}_{2}{ }^{\star}\right) . \mathrm{NO}_{2}{ }^{\star}$ changes back to the ground state while emitting electromagnetic radiation in the wavelength range $600-3000 \mathrm{~nm}$. A photomultiplicator tube that proportionally converts the intensity of luminescence into an electrical signal detects this chemiluminescence. The chemiluminescence technique is highly sensitive and NO can be detected in levels down to parts per trillion. ${ }^{319}$ It is rapid and easy to use which allows for on line measurements of exhaled NO. Other techniques that have been used to establish that $\mathrm{NO}$ is present in the exhaled breath of humans include mass spectroscopy and gas chromatography-mass spectroscopy. ${ }^{420}$

Several techniques have been used for collecting nasal air, the most commonly used method being to sample nasal air directly from one nostril. ${ }^{21}{ }^{22}$ Using the intrinsic flow of the chemiluminescence analyser or an external pump, air is aspirated from one nostril. The contralateral nostril is left open and the subject either holds his/her breath or breathes through the mouth. Air is thereby forced from one side of the nose to the other via the nasopharynx. To avoid contamination with NO from the lower airway the subject can be asked to exhale orally against a resistance in order to close the soft palate. ${ }^{23} 24$

Another suggested method of measuring nasal NO is to exhale through the nose after a 
Table 1 Nasal nitric oxide (NO): effects of disease

\begin{tabular}{llll}
\hline Disease & Age & Nasal NO & Reference \\
\hline Kartagener's syndrome & Children 2.5-12 yrs & Extremely low & 6 \\
Primary ciliary dyskinesia & Adults & Extremely low & 69 \\
Cystic fibrosis & Children $5-15$ yrs & Decreased & $11,31,32$ \\
Allergic rhinitis & Children 5-15 yrs & Unchanged & 11 \\
& Adults & Increased & $34-37$ \\
Acute sinusitis & Children & Decreased & 12 \\
Chronic sinusitis & Adults & Decreased & 33 \\
Common cold & Adults & Unchanged & 42 \\
Preeclampsia & Adults & Unchanged & 44 \\
\hline
\end{tabular}

full inhalation to vital capacity. The exhalation should be made at a constant flow rate. Following this procedure the subject then exhales through the mouth from full vital capacity at the same flow rate and the $\mathrm{NO}$ value from oral exhalation is subtracted from that measured during nasal exhalation (unpublished observation).

The optimal measurement technique for nasal NO is yet to be determined. A reliable and standardised method will allow for better comparisons of results between different laboratories. Attempts to standardise nasal NO measurements are in progress and recommendations for suitable sampling techniques will be given in the near future.

\section{Factors that influence nasal NO levels} MEASUREMENT TECHNIQUE

Since NO is continuously released into the nasal airways the concentration will be dependent on the flow rate by which the sample is aspirated..$^{21}$ Thus, nasal NO concentrations are higher at lower flow rates. It may therefore be preferable to express nasal $\mathrm{NO}$ as the output per time unit (e.g. $\mathrm{nl} / \mathrm{min}$ ) which has been shown to be relatively independent of flow rate. $^{25}$

PHYSIOLOGICAL FACTORS

In an early study nasal NO was measured in healthy subjects at different ages ranging from 0 to 70 years. ${ }^{9}$ Interestingly, nasal $\mathrm{NO}$ was already present at birth. In a later study Schedin et al found significant nasal NO levels in newborn infants including those delivered by caesarian section. ${ }^{26}$ In a study by Lundberg et al the same flow rate was used in all age groups and nasal NO was found to be much lower in newborn babies and in infants than in adults. However, if the nasal NO concentration from these data is calculated in relation to body weight, the concentration in children around 10 years of age is found to be approximately twice as high as that in newborn infants and adults. There is no evidence of sex differences in nasal NO, but variation in NO levels has not yet been studied in relation to the menstrual cycle. NO output in the nasal airways is acutely decreased by physical exercise. ${ }^{27-30}$ In the study by Lundberg et $a l^{27}$ direct measurement from one maxillary sinus showed an $83 \%$ reduction in sinus NO concentrations after five minutes of exercise. The decrease in nasal NO levels cannot be explained merely by dilution of nasal air due to changes in nasal cavity volume or increased ventilation. Instead, these changes in nasal NO have been attributed to a reduction

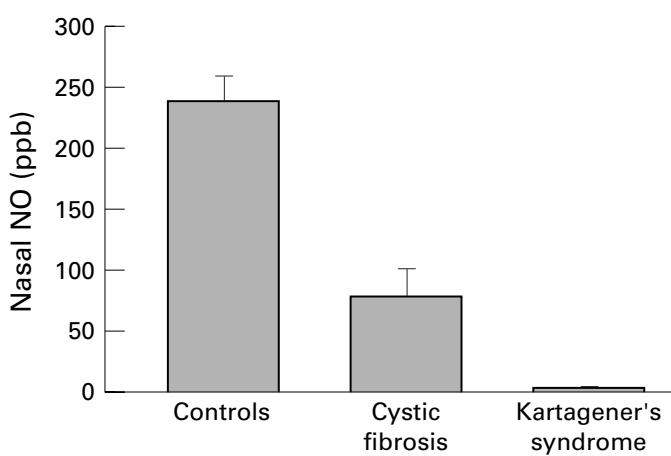

Figure 1 Steady state NO levels in air sampled directly from one nostril of healthy children, children with cystic fibrosis, and children with Kartagener's syndrome. Data from Lundberg. ${ }^{611}$

in the blood flow in the mucosa of the nasal airways with a concomitant decrease in substrate supply to the high rate producing NOS in the paranasal sinuses. ${ }^{27}$

DISEASES

The effects of various diseases on nasal NO levels are summarised in table 1 . In children with Kartagener's syndrome-a triad consisting of sinusitis, bronchiectasis, and situs inversus-nasal NO levels are extremely low compared with healthy, age matched controls. ${ }^{6}$ Nasal NO levels are also very low in patients with cystic fibrosis (fig 1). ${ }^{1131} 32$ Baraldi et al measured nasal NO in a group of children with acute sinusitis and found low nasal NO levels. ${ }^{12}$ In these patients nasal NO levels increased when the patients improved following treatment with antibiotics. Chronic sinusitis is associated with more than a $50 \%$ reduction in nasal NO levels. ${ }^{33}$ In general, the above studies indicate that nasal NO levels are lower in subjects with sinus disorders. It has not been established whether the low nasal NO levels in patients with sinus disorders are purely a result of altered passage of $\mathrm{NO}$ gas from the sinuses to the nasal cavity or whether the actual production of NO in the sinuses is decreased in these patients.

Whether or not nasal NO levels are altered in patients with rhinitis of different aetiology has not been fully established. Some groups have reported higher nasal NO levels in patients with allergic rhinitis. ${ }^{34-38}$ In one study nasal NO decreased following treatment with nasal topical steroids. ${ }^{35}$ Kirsten et al reported increased nasal NO levels after nasal challenge with endotoxin-containing swine confinement dust. ${ }^{39}$ In contrast, we found no alterations in nasal NO levels in a group of children with perennial rhinitis. ${ }^{11}$ The reasons for the discrepancies between the studies are not clear. One might speculate that iNOS is upregulated in the nose during rhinitis, ${ }^{40}$ as is the case in the lower airways in asthma. ${ }^{41}$ This would explain the higher levels of NO reported in some studies. On the other hand, the swelling of the nasal mucosa present during rhinitis might also lead to partial blockage of the sinus ostia which would result in reduced passage of sinus NO to the nasal cavity where it is measured.

Eccles et al measured nasal NO levels in patients with a common cold but failed to see 
Table 2 Nasal nitric oxide (NO): effects of drugs, cigarette smoke and physical exercise

\begin{tabular}{llll}
\hline Drug & Administration & Nasal NO & Reference \\
\hline Glucocorticoids & Nasal instillation & Unchanged & 6 \\
Healthy subjects & Systemic & Unchanged & 16 \\
& Nasal instillation & Decreased & 35 \\
Allergic rhinitis & Nasal instillation & Minor decrease & 9,45 \\
NO synthase inhibitors & Systemic & Decreased & 46 \\
& Nasal spray & Unchanged & 45 \\
L-arginine & Nasal spray & Increased & 69 \\
& Systemic & Increased & 16,44 \\
Histamine, capcaisin & Nasal spray & Unchanged & 45 \\
Alpha-2 agonists & Nasal spray & Decreased & $37,42,45$ \\
Cigarette smoke & & Decreased & 7,47 \\
Antibiotics & Systemic & Unchanged & 6 \\
Physical exercise & & Decreased & $27-30$ \\
\hline
\end{tabular}

any differences compared with healthy controls. ${ }^{42}$ In another study on experimental human influenza nasal NO levels decreased slightly during the symptomatic period. ${ }^{43}$

A general NO deficiency has been implicated in the pathogenesis of pre-eclampsia, a disease of pregnancy characterised by oedema, hypertension, and proteinuria. However, in a recent study we found similar nasal levels of $\mathrm{NO}$ in patients and healthy pregnant controls. ${ }^{44}$

DRUGS

The effects of various drugs on nasal $\mathrm{NO}$ are summarised in table 2. Early reports on the effect of topical glucocorticoids showed no effect of these drugs on nasal NO levels. ${ }^{6}$ In fact, not even systemic steroids in high doses seemed to alter nasal NO levels. ${ }^{16}$ Gerlach et al suggested that bacteria in the nasopharynx induced nasal NO release. ${ }^{7}$ However, we found no effects of systemic antibiotic treatment on nasal NO levels. ${ }^{6}$ Moreover, as already mentioned, newborn babies delivered by caesarian section had high nasal NO levels. ${ }^{26}$ Nasal NO release therefore seems to be independent of bacteria normally present in the nasopharynx.

Topical nasal decongestants such as oxymetazoline have been shown to decrease nasal NO levels. ${ }^{37} 45$ The reason for this is not clear but it has been suggested that the decrease in nasal/sinus blood flow induced by these drugs leads to a reduction of substrate supply to the high producing iNOS in the sinuses. ${ }^{45}$ Indeed, substrate supply seems to be of importance for nasal/sinus NO release since intravenous administration of L-arginine resulted in an increase in nasal NO levels. ${ }^{16}$

Rinder et al looked at the effects of histamine and capsaicin on nasal NO levels but found no effects of these drugs. ${ }^{45}$

The NO synthase inhibitor $\mathrm{N}^{\mathrm{G}}$-nitro-Larginine methyl ester (L-NAME) has only minor effects on nasal NO levels when administered locally in the nose. ${ }^{9}{ }^{45}$ In contrast, Albert et al found a substantial decrease in nasal NO levels following intravenous administration of $\mathrm{N}^{\mathrm{G}}$-monomethyl-L-arginine (L-NMMA), another NOS inhibitor. ${ }^{46}$ This fits well with the theory that the paranasal sinuses are the main source of NO in the nasal airways. Thus, NOS inhibitors probably penetrate poorly into the sinuses if administered locally in the nose whereas, if given intravenously, these drugs will reach the sinus mucosa.
Nasal NO levels have been reported to be lower in cigarette smokers ${ }^{77}$ but increase one week after smoking cessation. ${ }^{47}$

\section{Animal models}

Schedin et al have measured nasal NO in a large number of species and found high levels only in primates and in elephants. ${ }^{13}$ Lewandowski et al recently measured nasal $\mathrm{NO}$ in baboons and found only low levels. ${ }^{14}$ Interestingly, baboons are the only mammal known to lack paranasal sinuses. Because of the large variation in nasal $\mathrm{NO}$ output between different species, caution should be taken when comparing data from animal models with results from studies in humans.

\section{Role of nasal NO}

Although the exact role of nasal NO is far from clear, it is reasonable to believe that this pluripotent gas is involved in a variety of physiological as well as pathophysiological events in the airways.

\section{HOST DEFENCE}

Among the various biological properties of $\mathrm{NO}$ are its effects on the growth of various pathogens including bacteria, fungi, and viruses. ${ }^{2}{ }^{48} \mathrm{NO}$ produced by white blood cells is thought to be important in the killing of certain micro-organisms by these cells. Furthermore, some bacteria are sensitive to authentic NO gas in concentrations as low as 100 parts per billion (ppb). ${ }^{49}$ The fact that local NO concentrations in the nasal airways may be several hundred times higher than this supports the notion that $\mathrm{NO}$ is involved in local host defence in the upper airways. Local concentrations in the paranasal sinuses can reach 30000 ppb. ${ }^{9}$ We have suggested that this NO may help to keep the sinuses sterile under normal conditions. One might speculate that very low nasal NO levels in patients with Kartagener's syndrome or cystic fibrosis ${ }^{8}$ contribute to the increased susceptibility to airway infections in these patients. If this is correct, stimulation of endogenous NO production could increase the resistance to airway infections in patients with low nasal NO levels as suggested earlier. ${ }^{6}$ It is not clear whether NO itself acts directly on micro-organisms or whether it combines with other components to yield other reactive nitrogen intermediates that are toxic. ${ }^{50}$

Besides acting directly on micro-organisms, NO may also contribute to local host defence by stimulating ciliary motility. ${ }^{51}$ A study by Runer et al showed that application of an NO donor in the nasal mucosa of humans did, indeed, cause an increase in ciliary beat frequency. ${ }^{52}$ Furthermore, the same group has shown that low levels of nasal NO correlate with impaired mucociliary function in the human upper airways. ${ }^{53}$

\section{INFLAMMATION}

NO synthesis is clearly enhanced locally at sites of inflammation. ${ }^{54}$ This has been described not only in asthma ${ }^{5}$ but also in, for example, inflammatory bowel disease ${ }^{55} 56$ and cystitis. ${ }^{57}$ However, the role of $\mathrm{NO}$ in inflammation is far 
from certain. Some studies indicate a harmful role for NO during inflammation, whereas others indicate the opposite. Possible proinflammatory actions of $\mathrm{NO}$ include activation of enzymes such as cyclo-oxygenase or metalloproteases. Moreover, peroxynitrite, which is formed from the reaction of $\mathrm{NO}$ with superoxide, can exert toxic effects to tissues. ${ }^{3658}$ The possible harmful effects of $\mathrm{NO}$ have been attributed to the large amounts of this gas produced by iNOS during inflammation. However, the recent finding of a constantly expressed iNOS in the nasal airways complicates this picture since it clearly demonstrates that the sole expression of iNOS and the subsequent increased production of $\mathrm{NO}$ is not associated with tissue damage. On the contrary, iNOS in the upper airways may serve important protective functions as discussed above. Furthermore, in an animal model of colon inflammation McCafferty et al have shown that mice lacking the iNOS gene develop a much more severe inflammation than wild type mice. ${ }^{59}$

NASAL NO AS AN AIRBORNE MESSENGER

The effect of inhaled exogenous $\mathrm{NO}$ is currently being investigated in large scale clinical trials in patients with pulmonary hypertension and/or adult respiratory distress syndrome and its clinical use in newborn children with persistent pulmonary hypertension has been reported. ${ }^{60}$ Several investigators have found clear effects on arterial oxygenation and pulmonary arterial pressure using concentrations of inhaled NO as low as $10-100 \mathrm{ppb}^{.6162}$ Interestingly, during normal breathing endogenous NO is inhaled at concentrations (approximately $100 \mathrm{ppb}$ ) which are known to have vasodilating effects in the pulmonary circulation. ${ }^{6}$ We have shown that nasal breathing reduces pulmonary vascular resistance and improves arterial oxygenation compared with oral breathing in subjects without lung disease (fig 2). ${ }^{86364}$ The addition of 100 ppb NO during oral breathing mimicked the effect of nasal breathing while moistened air during oral breathing had no effect. ${ }^{63}$ Intubated patients are deprived of the natural inhalation of endogenous upper airway NO. Supplementation of nasal air to intubated patients treated

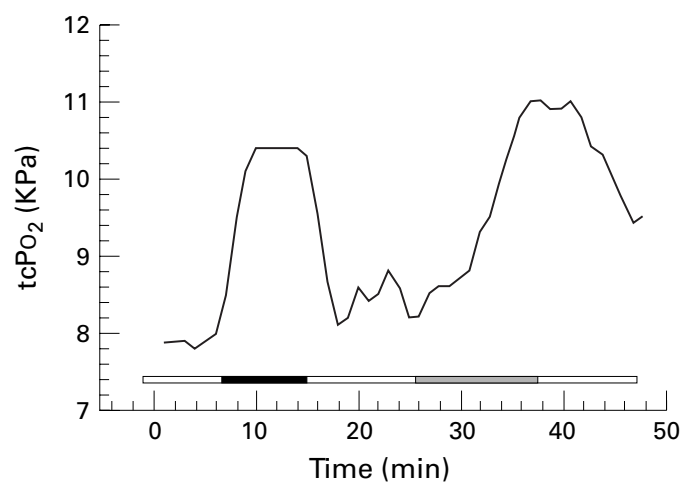

Figure 2 Representative tracing showing transcutaneous oxygen tension in a healthy subject during periods of oral breathing (empty bars), nasal breathing (filled bar), or oral breathing of air containing exogenous $\mathrm{NO}$ at a concentration of $100 \mathrm{ppb}$ (hatched bar). Reprinted from Lundberg et al ${ }^{63}$ with permission. with a ventilator also improves arterial oxygenation and reduces pulmonary vascular resistance. ${ }^{65}$ Moreover, Pinsky et al have shown that the hospital pressurised air may contain NO levels similar to those described above (6-500 ppb) which may consequently have effects on arterial oxygenation and pulmonary arterial pressure in mechanically ventilated patients. ${ }^{66}$ In a recent study nostril widening with a nasal tape improved arterial oxygenation in spontaneously breathing patients, probably by enhancing ventilation through the nasal airways thereby increasing the delivery of NO from the nasal airways to the lungs. ${ }^{68}$

These results show that NO derived from the upper airways is capable of improving oxygen uptake and reducing pulmonary vascular resistance (fig 3). It is tempting to speculate that the production of $\mathrm{NO}$ in the paranasal sinuses has the purpose of modulating lung function in humans. Since NO is produced above the bifurcation of the large airways this vasodilating gas will only affect pulmonary vessels in contact with ventilated alveoli, thereby improving ventilation/perfusion matching. This new physiological principle of $\mathrm{NO}$ as an airborne mediator may extend beyond vasodilation. Further studies will reveal whether inhalation of endogenous NO also has antimicrobial effects, upregulates ciliary motility, and inhibits platelet aggregation. If this is true the well known complications associated with long term intubation and ventilation, such as ciliary dysfunction and bacterial infections, may partly be explained by the lack of NO due to disruption of the natural low dose flushing of the lower airways by self-inhalation of endogenous NO from the upper airways. ${ }^{69}$

Clinical value of nasal NO measurements

Measurements of nasal NO levels are attractive since they are completely non-invasive and can easily be performed even in small children. The

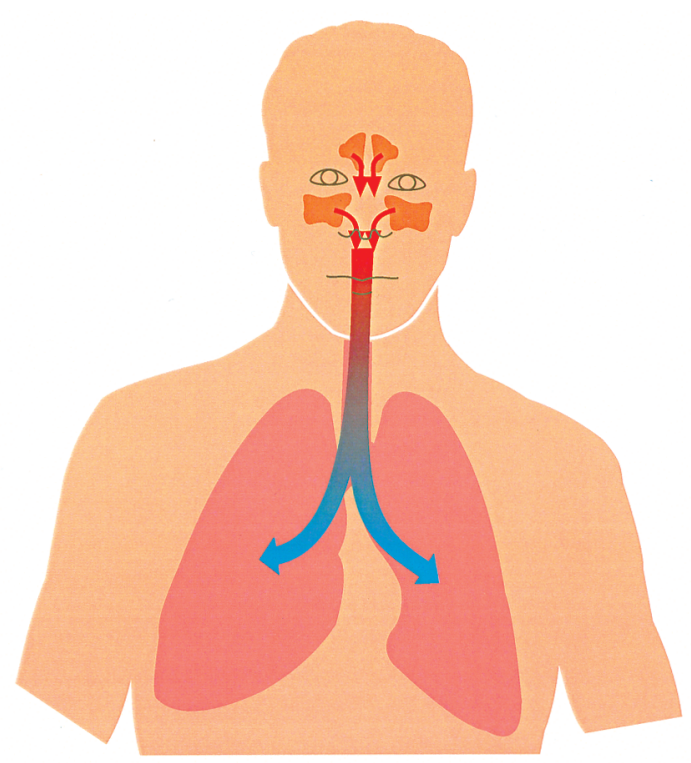

Figure 3 Nitric oxide (NO) is released in the nasal airways in humans. During inspiration through the nose this NO will follow the airstream to the lower airways and the lungs. Nasally derived NO has been shown to increase arterial oxygen tension and reduce pulmonary vascular resistance, thereby acting as an airborne messenger. 
finding of low nasal NO levels in patients with chronic sinus disorders such as Kartagener's syndrome and cystic fibrosis are interesting. ${ }^{8}$ It is tempting to speculate that nasal $\mathrm{NO}$ measurements may be used to facilitate early diagnosis of these two respiratory disorders.

The possible usefulness of nasal NO measurements in the diagnosis and treatment monitoring of allergic rhinitis clearly needs to be further evaluated. If nasal $\mathrm{NO}$ is a reliable marker of local inflammation, this simple test may be used to monitor patients with rhinitis of different aetiology.

As mentioned above, intubated patients are deprived of inhaling the NO produced in the nasal airways. Considering the possible beneficial effects of nasal $\mathrm{NO}$ on pulmonary function, it is possible that replacement of nasal NO would be of benefit to patients being ventilated with an endotracheal tube.

It is reasonable to believe that drugs that affect endogenous NO synthesis will be used clinically in the future. These drugs may either facilitate or decrease NO production by, for example, interfering with different NOS. Measurement of nasal NO could be an interesting non-invasive means of monitoring the effects of such drugs and of optimising the dosage. The non-selective NOS inhibitor L-NMMA is currently being investigated for use in patients with sepsis. Interestingly, this inhibitor, when administered intravenously, leads to a dose dependent decrease in nasal NO levels. ${ }^{46}$ Conversely, systemic administration of L-arginine increases nasal NO levels. ${ }^{16}$

\section{Summary and future directions}

Large amounts of NO are constantly being released in the nasal airways of humans and the paranasal sinuses seem to be the major source of this NO. Nasal NO may have important local as well as distal effects in, for example, host defence and regulation of pulmonary function.

The measurement of nasal NO levels may be helpful in further exploring the physiological and pathophysiological roles of this gas in the airways. Moreover, this simple test may be clinically useful in the diagnosis and treatment monitoring of certain respiratory disorders. To evaluate fully the potential of nasal NO measurements in the clinical setting it is important that we develop a standardised and reliable technique. We also need to know more about the different factors that affect nasal NO levels.

1 Moncada S, Higgs A. The L-arginine-nitric oxide pathway. $N$ Engl f Med 1993;329:2002-12.

2 Nathan C, Xie Q-W. Nitric oxide synthases: roles, tolls, and controls. Cell 1994;78:915-8.

3 Archer S. Measurement of nitric oxide in biological models. FASEB ₹ 1993;7:349-60.

4 Gustafsson LE, Leone AM, Persson MG, et al. Endogenous nitric oxide is present in the exhaled air of rabbits, guinea pigs and humans. Biochem Biophys Res Commun 1991;181: $852-7$.

5 Alving K, Weitzberg E, Lundberg JM. Increased amounts of nitric oxide in exhaled air of asthmatics. Eur Respir F 1993; 6:1368-70.

6 Lundberg JON, Weitzberg E, Nordvall SL, et al. Primarily undberg JON, Weitzberg E, Nordvall SL, et al. Primarily Kartagener's syndrome. Eur Respir f 1994;7:1501-4.

7 Gerlach H, Rossaint R, Pappert D, et al. Autoinhalation of nitric oxide after endogenous synthesis in nasopharynx. Lancet 1994;343:518-9.
8 Lundberg JON. Airborne nitric oxide: inflammatory marker and aerocrine messenger in man. Acta Physiol Scand 1996; 157:1-27.

9 Lundberg JON, Farkas-Szallasi T, Weitzberg E, et al. High nitric oxide production in human paranasal sinuses. Nature Med 1995;1:370-3.

10 Lundberg JON, Rinder J, Weitzberg E, et al. Nasally exhaled nitric oxide in humans originates mainly in the paranasal sinuses. Acta Physiol Scand 1994;152:431-2.

11 Lundberg JON, Nordvall SL, Weitzberg E, et al. Exhaled NO in pediatric asthma and cystic fibrosis. Arch Dis Child 1996;75:323-6.

12 Baraldi E, Azzolin N, Biban P, et al. Effect of antibiotic therapy on nasal nitric oxide concentration in children with acute sinusitis. Am f Respir Crit Care Med 1997;155:1680-

13 Schedin U, Roken B, Nyman G, et al. Endogenous nitric oxide in the airways of different animal species. Acta Anaesthesiol Scand 1997;41:1133-41.

14 Lewandowski K, Busch T, Lohbrunner H, et al. Low nitric oxide concentrations in exhaled gas and nasal airways of mammals without paranasal sinuses. F Appl Physiol 1998;85:405-10

15 Geller DA, Lowenstein CJ, Shapiro RA, et al. Molecular cloning and expression of inducible nitric oxide synthase from hepatocytes. Proc Natl Acad Sci USA 1993;90:3491-

16 Lundberg JON, Weitzberg E, Rinder J, et al. Calciumindependent and steroid-resistant nitric oxide synthase activity in human paranasal sinus mucosa. Eur Respir $f$ 1996; 9:1344-7.

17 Ramis I, Lorente J, Roselló-Catafau J, et al. Differential activity of nitric oxide synthase in human nasal mucosa and polyps. Eur Respir F 1996;9:202-6.

18 Watkins D, Lewis R, Basclain K, et al. Expression and localization of the inducible isoform of nitric oxide synthase in nasal polyp epithelium. Clin Exp Allergy 1998;28:211-9.

19 Fontijin A, Sabadell A, Ronco R. Homogenous chemiluminescent measurement of nitric oxide with ozone: implications for continuous selective monitoring of gaseous air tions for continuous selective monitorin

20 Leone AM, Gustavsson LE, Francis PL, et al. Nitric oxide is present in exhaled breath in humans: direct GC-MS confirmation. Biochem Biophys Res Commun 1994;201:883-

21 Lundberg JON, Weitzberg E, Lundberg JM, et al. Nitric oxide in exhaled air. Eur Respir f 1996;9:2671-80.

22 Kharitonov S, Alving K, Barnes PJ. Exhaled and nasal nitric oxide measurements: recommendations. Eur Respir 71997 10:1683-93.

23 Kimberly B, Nejadnik B, Giraud G, et al. Nasal contribution to exhaled nitric oxide at rest and during breathholding. Am 7 Respir Crit Care Med 1996;153:829-36.

24 Silkoff P, McClean P, Slutsky A, et al. Marked flowdependence of exhaled nitric oxide using a new technique to exclude nasal nitric oxide. Am f Respir Crit Care Med 1997; 155:260-7

25 Dubois A, Douglas J, Stitt J, et al. Production and absorption of nitric oxide in the nose. F Appl Physiol 1998, 84:1217-24.

26 Schedin U, Gustafsson LE, Herin P, et al. Endogenous nitric oxide in the upper airways of healthy new-born infants. Oxide in the upper airways

27 Lundberg JON, Rinder J, Weitzberg E, et al. Heavy physical exercise acutely decreases nitric oxide levels in the nasal airways in man. Acta Physiol Scand 1996;159:51-7.

28 Imada M, Iwamoto J, Nonaka S, et al. Measurement of nitric oxide in human nasal airway. Eur Respir 7 1996;9:556-9.

29 Phillips C, Giraud G, Holden W. Exhaled nitric oxide during exercise: site of release and modulation by ventilation and flow. F Appl Physiol 1996;80:1865-71.

30 Yasuda Y, Itoh T, Miyamura M, et al. Comparisons of exhaled nitric oxide and cardiorespiratory indices between nasal and oral breathing during submaximal exercise in humans. Fapanese f Physiol 1997;47:465-70.

31 Balfour-Lynn I, Laverty A, Dinwiddle R. Reduced upper airway nitric oxide in cystic fibrosis. Arch Dis Child airway nitric oxide.

32 Dotsch J, Demirakca S, Terbrack H, et al. Airway nitric oxide in asthmatic children and patients with cystic fibrosis. Eur Respir f 1996;9:2537-40.

33 Lindberg S, Cervin A, Runer T. Nitric oxide (NO) production in the upper airways is decreased in chronic sinusitis. Acta Oto-Laryngol 1997;117:113-7.

34 Martin U, Bryden K, Devoy M, et al. Increased levels of exhaled nitric oxide during nasal and oral breathing in subjects with seasonal rhinitis. F Allergy Clin Immunol 1996;97: jects with

35 Kharitonov S, Rajakulasingam K, O'Connor B, et al. Nasal nitric oxide is increased in patients with asthma and allergic rhinitis and may be modulated by nasal glucocorticosteroids. F Allergy Clin Immunol 1997;99:58-64.

36 Sato M, Fukuyama N, Sakai M, et al. Increased nitric oxide in nasal lavage fluid and nitrotyrosine formation in nasal mucosa: indices for severe perennial nasal allergy. Clin Exp Allergy 1998;28:597-605.

37 Arnal J, Didier A, Rami J, et al. Nasal nitric oxide is increased in allergic rhinitis. Clin Exp Allergy 1997;27:34750.

38 Adisesh L, Kharitonov S, Yates D, et al. Exhaled and nasal nitric oxide is increased in laboratory animal allergy. Clin Exp Allergy 1998;28:876-80. 
39 Kirsten A, Jorres R, Kirsten D, et al. Effect of a nasal challenge with endotoxin-containing swine confinemen dust on nasal nitric oxide production. Eur F Med Res 1997; 2:335-9.

40 Furukawa K, Harrison DG, Saleh D, et al. Expression of nitric oxide synthase in the human nasal mucosa. Am Respir Crit Care Med 1996;153:847-50.

41 Hamid Q, Springall DR, Riveros-Moreno V, et al. Induction of nitric oxide synthase in asthma. Lancet 1993;342:1510 3 .

42 Ferguson E, Eccles R. Changes in nasal nitric oxide concentration associated with symptoms of common cold and treatment with a topical nasal decongestant. Acta OtoLaryngol 1997;117:614-7.

43 Murphy A, Plattmills T, Lobo M, et al. Respiratory nitric oxide levels in experimental human influenza. Chest 1998; 114:452-6.

44 Grunewald C, Carlström K, Kumelin G, et al. Exhaled oral and nasal nitric oxide during L-arginine infusion in preeclampsia. Gynecol Obstet Invest 1998;46:232-7.

45 Rinder J, Lundberg JON, Änggård A, et al. Effects of topica nasal decongestants, L-arginine and nitric oxide synthase inhibition on nasal cavity nitric oxide levels and nasal cavity volume in man. Am F Rhinol 1996;10:399-408.

46 Albert J, Schedin U, Lindqvist M, et al. Blockade of endogenous nitric oxide production results in moderate hypertension, reducing sympathetic activity and shortening bleeding time in healthy volunteers. Acta Anaesthesiol Scand 1997;41:1104-13.

47 Robbins R, Millatmal T, Lassi K, et al. Smoking cessation is associated with an increase in exhaled nitric oxide. Chest 1997;112:313-8

48 Sanders S, Siekierski E, Porter J, et al. Nitric oxide inhibits rhinovirus-induced cytokine production and viral replication in a human respiratory epithelial cell line. F Virol 1998 72:934-42

49 Mancinelli RL, McKay CP. Effects of nitric oxide and nitrogen dioxide on bacterial growth. Appl Environ Microbiol 1983;46:198-202.

50 Xie Q-W, Nathan C. The high output nitric oxide pathway: role and regulation. F Leucocyte Biol 1994;56:576-82.

51 Jain B, Rubenstein I, Robbins RA, et al. Modulation of airway epithelial cell ciliary beat frequency by nitric oxide. Biochem Biophys Res Commun 1993;191:83-8.

52 Runer T, Cervin A, Lindberg S, et al. Nitric oxide is a regulator of mucociliary activity in the upper respiratory tract. Otolaryngol Head Neck Surg 1998;119:278-87.

53 Lindberg S, Cervin A, Runer T. Low levels of nasal nitric oxide (NO) correlate to impaired mucociliary function in the upper airways. Acta Oto-Laryngol 1997;117:728-34.

54 Lundberg JON, Lundberg JM, Alving K, et al. Nitric oxide and inflammation: the answer is blowing in the wind. Nature Med 1997;3:30-1.
55 Lundberg JON, Hellström PM, Lundberg JM, et al. Nitric oxide in ulcerative colitis. Lancet 1995;345:449.

56 Lundberg JON, Hellström PM, Lundberg JM, et al. Greatly increased luminal nitric oxide in ulcerative colitis. Lancet 1994;344:1673-74.

57 Lundberg JON, Adofsson J, Jansson O, et al. Elevated nitric oxide in the urinary bladder in infectious and noninfectious cystitis. Urology 1996;48:700-2.

58 Beckman JS, Beckman TW, Chen J, et al. Apparent hydroxyl radical production by peroxinitrite: implications for endothelial cell injury from nitric oxide. Proc Natl Acad Sci USA 1990;87:1620-4

59 McCafferty DM, Mudgett JS, Swain MG, et al. Inducible nitric oxide synthase plays a critical role in resolving intestinal inflammation. Gastroenterology 1997;112:1022-7.

60 Davidson D, Barefield E, Kattwinkel J, et al. Inhaled nitric oxide for the early treatment of persistent pulmonary
hypertension of the term new-born: a randomized, doublehypertension of the term new-born: a randomized, double-
masked, placebo-controlled, dose-response, multicenter masked, placebo-controlled, dose-response, multicenter study. The

61 Puybasset L, Rouby J, Mourgeon E, et al. Inhaled nitric oxide in acute respiratory failure: dose-response curves. Intensive Care Med 1994;20:319-27.

62 Gerlach H, Rossaint R, Pappert D, et al. Time-course and dose-response of nitric oxide inhalation for systemic oxygenation and pulmonary hypertension in patients with oxygenation and pulmonary hypertension in patients with 23:499-502.

63 Lundberg JON, Settergren G, Gelinder S, et al. Inhalation of nasally derived nitric oxide modulates pulmonary function in humans. Acta Physiol Scand 1996;158:343-7.

64 Settergren G, Angelin B, Liska J, et al. Lower pulmonary vascular resistance during nasal breathing: modulation by nitric oxide from the paranasal sinuses. Acta Physiol Scand 1998;163:235-9.

65 Lundberg JON, Lundberg JM, Settergren G, et al. Nitric oxide, produced in the upper airways, may act in an aerocrine fashion to enhance pulmonary oxygen uptake in humans. Acta Physiol Scand 1995;155:467-8.

66 Lee K, Tan P, Rico P, et al. Low levels of nitric oxide as contaminant in hospital compressed air: physiologic significance? Crit Care Med 1997;25:1143-6.

67 Pinsky M, Genc F, Lee K, et al. Contamination of hospital compressed air with nitric oxide: unwitting replacement therapy. Chest 1997;111:1759-63.

68 Herulf M, Lundberg JON, Weitzberg E. Nostril widening and arterial oxygenation. Respir Med 1999;93:134-5.

69 Loukides S, Kharitonov S, Wodehouse T, et al. Effect of arginine on mucociliary function in primary ciliary dyskinesia. Lancet 1998;352:371-2. 